\title{
Drug information update: agomelatine
}

\author{
Daniel Whiting, ${ }^{1}$ Philip J. Cowen ${ }^{1}$
}

The Psychiatrist (2013), 37, 356-358, doi: 10.1192/pb.bp.113.043505

${ }^{1}$ University Department of Psychiatry, Warneford Hospital, Oxford

Correspondence to Philip Cowen (phil.cowen@psych.ox.ac.uk)

First received 24 Mar 2013, final revision 3 Apr 2013, accepted 4 Apr 2013
Summary Agomelatine is a new antidepressant, licensed for the treatment of unipolar major depression, with a mode of action that combines activation of melatonin receptors with blockade of $5-\mathrm{HT}_{2} \mathrm{C}$ receptors. Agomelatine is notable for its short duration of action in the body and modest side-effect burden; however, a number of theoretical and practical challenges have limited its adoption into mainstream treatment in the UK. Current meta-analyses show marginal clinical benefits of agomelatine relative to placebo and an association with occasional increases in liver transaminases. Theoretically it is not clear whether agomelatine does block brain $5-\mathrm{HT}_{2 \mathrm{C}}$ receptors in humans at therapeutic doses and the optimum daily timing of administration in depression has not been clearly established. However, agomelatine's novel mode of action justifies further study, perhaps with the eventual aim of matching its use in depression to patients with specific disturbances in circadian rhythm.

Declaration of interest P.J.C. has been a paid member of advisory boards for Eli Lilly, Lundbeck and Servier.
The melatonin analogue, agomelatine, is the first antidepressant approved by the European Medicines Agency which is not monoaminergic. Agomelatine is proposed to act as an agonist at melatonin (MT1 and MT2) receptors, and an antagonist at $5-\mathrm{HT}_{2 \mathrm{C}}$ receptors. Controlled studies have suggested favourable efficacy and tolerability profiles; however, agomelatine is not without its controversies, with recent meta-analyses showing only marginal advantages over placebo. It is nevertheless an intriguing agent pharmacologically and here we provide a synopsis of its proposed mechanisms of action, indications and side-effects in the treatment of unipolar major depression.

\section{Mechanism of action}

Disturbances of the sleep-wake cycle and diurnal variation in mood are common symptoms of depression. A role for abnormalities in circadian rhythm in the pathophysiology of depression therefore makes intuitive sense, and has long been explored in treatments such as sleep deprivation and light therapy. ${ }^{1}$ However, it is not yet clearly established whether there is a consistent circadian 'phase-shift' in patients with major depression or (as seems more likely in this heterogeneous disorder) that some, but not all, patients have variable kinds of circadian disturbances. For example, the pineal hormone melatonin is a reliable marker of circadian rhythm and the activity of the circadian 'oscillator' in the suprachiasmatic nucleus (SCN). However, despite many studies it is still not clear whether there are abnormalities in the amplitude and timing of melatonin secretion in patients with depression. ${ }^{2}$ Nevertheless, it is known that in both animals and humans appropriately timed administration of melatonin has the capacity to shift circadian rhythms, and melatonin is used for this purpose, for example, in delayed sleep-phase syndrome. ${ }^{3}$

Agomelatine ( $N$-[2-(7-methoxy-1-napthyl)ethyl]acetamide) is a melatonin analogue, binding with high affinity to MT1 and MT2 G-protein coupled receptors. ${ }^{4}$ Melatonin receptors are expressed in numerous regulatory areas of the brain, including the $\mathrm{SCN}^{5}$ MTl activation regulates amplitude of circadian rhythmicity via inhibition of SCN neurons, and MT2 activation is involved in phase-shifts and entrainment of the rhythm. ${ }^{6}$ Additionally, and distinctly from melatonin, agomelatine is a competitive antagonist at $5-\mathrm{HT}_{2 \mathrm{C}}$ and $5-\mathrm{HT}_{2 \mathrm{~B}}$ receptors, and in animal studies, $5-\mathrm{HT}_{2 \mathrm{C}}$ antagonism has the ability to increase noradrenaline and dopamine release in the prefrontal cortex. ${ }^{4}$

The antidepressant effects of agomelatine are claimed to derive from a synergism between its melatonergic and $5-\mathrm{HT}_{2 \mathrm{C}}$ actions. Thus results from animal experimental studies of behavioural models of depression show that melatonin or $5-\mathrm{HT}_{2 \mathrm{C}}$ antagonists alone are insufficient to produce the full antidepressant activity of agomelatine. ${ }^{7}$ Similarly, agomelatine produces an increase in gene expression of brain-derived neurotrophic factor (BDNF) in prefrontal cortex that is not reproduced with melatonin or 5- $\mathrm{HT}_{2 \mathrm{C}}$ antagonism alone. ${ }^{8}$ Brain-derived neurotrophic factor has been implicated in depression and the response to antidepressant therapy. ${ }^{9}$

However, the affinity of agomelatine for $5-\mathrm{HT}_{2 \mathrm{C}}$ receptors is significantly lower than its ability to bind melatonin receptors. ${ }^{4}$ It is therefore worth noting that there is currently no evidence that at clinically used doses agomelatine blocks brain $5-\mathrm{HT}_{2 \mathrm{C}}$ receptors in humans and 
in fact some evidence to the contrary. ${ }^{10}$ In contrast, the melatonergic action of agomelatine has been supported by small healthy volunteer studies in humans which indicate that agomelatine can phase-alter the timing of circadian rhythms. For example, a double-blind placebo study of eight healthy young men showed that agomelatine given at $18.00 \mathrm{~h}$ produced phase advancement as demonstrated by an earlier drop in core body temperature equivalent to melatonin administration. ${ }^{11}$ In a group of older men, 15 days of agomelatine administered at $18.30 \mathrm{~h}$ phase-advanced the drop in core body temperature by $1.5-2 \mathrm{~h}$, but without significantly affecting any sleep parameters including rapid eye movement (REM) sleep. ${ }^{12}$

\section{Dose and pharmacokinetics}

The starting dose of agomelatine is $25 \mathrm{mg}$ taken at 'bedtime', increasing to $50 \mathrm{mg}$ if indicated after 2 weeks. ${ }^{13}$ Agomelatine is well absorbed after oral administration but subject to extensive first-pass metabolism, which causes significant variation in drug plasma levels between individuals. Peak plasma levels occur within $1-2 \mathrm{~h}$ after ingestion and the terminal half-life of the drug is also between 1 and $2 \mathrm{~h}$. Therefore, accumulation of agomelatine does not occur. Agomelatine is metabolised in the liver by CYP1A2 and should therefore not be given with potent CYP1A2 inhibitors such as ciprofloxacin and fluvoxamine. Caution is recommended when using agomelatine with moderate CYP1A2 inhibitors such as propranolol and oestrogens. Conversely, smoking induces CYP1A2 activity and can lower agomelatine levels (www.medicines.org.uk/ EMC/medicine/21830/SPC/Valdoxan).

\section{Indications}

Agomelatine is licensed by the European Medicines Agency for the treatment of major depressive episodes in adults. There is currently insufficient randomised trial evidence to consider agomelatine in treatment-resistant depression or in bipolar depression, although some open case series are available.

\section{Clinical effects and efficacy}

Although narrative reviews of the efficacy of agomelatine emphasise its superior efficacy relative to placebo and certain other antidepressants such as sertraline and fluoxetine, ${ }^{14}$ formal meta-analyses have found these effects to be less convincing ${ }^{15,16}$ and of uncertain clinical significance. For example, Singh et $a l^{15}$ examined 9 double-blind randomised controlled trials of 3943 patients with major depression in which agomelatine was compared with either other antidepressants or placebo. In pooled analyses, agomelatine was significantly but marginally better than placebo (standardised mean difference in depression rating scales of -0.26). Data from five trials on 1698 patients showed again marginal superiority of agomelatine over the aggregated results of other antidepressants (fluoxetine, paroxetine, sertraline and venlafaxine) but not over any of the other agents examined individually. However, clear separation from placebo is not a problem unique to agomelatine among new antidepressants, perhaps partly because of the methodological difficulties involved in placebo-controlled, regulatory trials. ${ }^{17}$

In one study, agomelatine appeared effective in the maintenance treatment of depression, although in two other studies a positive effect could not be demonstrated. ${ }^{14}$

\section{Side-effects}

In controlled trials, the level of side-effects of agomelatine has generally been similar to placebo, with the exception of increased rates of nausea, diarrhoea and dizziness. ${ }^{18}$ Side-effects in the British National Formulary that are reported as 'common' also include constipation, headache, drowsiness, insomnia, fatigue, back pain and sweating. ${ }^{13}$ Agomelatine appears less likely to cause sexual side-effects than selective serotonin reuptake inhibitors (SSRIs) and serotonin-noradrenaline reuptake inhibitors (SNRIs), with studies suggesting that agomelatine has similar rates of emergent sexual dysfunction to placebo and significantly lower rates than drugs such as venlafaxine. ${ }^{19}$

The most problematic side-effect of agomelatine is its association with abnormal liver function tests, and jaundice and hepatitis have also been reported. Rates of elevation of liver transaminases more than three times normal are seen in about $4.5 \%$ of patients taking $50 \mathrm{mg}$ daily. ${ }^{18}$ Liver function tests are therefore required prior to initiation of agomelatine treatment and then after 3, 6, 12 and 24 weeks; agomelatine is contraindicated in people with hepatic impairment.

\section{Conclusions}

Agomelatine is an interesting antidepressant with a novel mode of action. More data are needed on the clinical effectiveness of agomelatine in real-world conditions, particularly in relation to other available antidepressant drugs. It would also be useful to have more data about the utility of agomelatine in patients with treatment-resistant depression, either as a sole antidepressant or as an augmenting agent. In an open study, Sparshatt et $a l^{20}$ followed 110 patients treated in secondary care with agomelatine; the majority $(75 \%)$ had failed to respond or had relapsed while taking current antidepressant treatment. In a fifth of the patients, agomelatine was added to ongoing antidepressant treatment. About $60 \%$ of the total group continued agomelatine up until 12 weeks, which was the primary outcome measure; the majority of discontinuations were for lack of efficacy. Just under half the patients showed an improvement on the Clinical Global Impression rating scale. Assessment of the effectiveness of agomelatine in this hard-to-treat patient group is difficult from these data but the authors concluded that the fact that the majority of patients continued agomelatine treatment for at least 12 weeks was encouraging.

At present, the main place for agomelatine is probably in patients with depression who are unable to tolerate conventional antidepressants, particularly if sexual dysfunction is a problem. Because of its mode of action, it is possible that agomelatine could be most effective in patients 
where the depressive syndrome is associated with abnormal circadian rhythm. Identifying such patients specifically would require a carefully targeted clinical assessment and probably the use of laboratory measures such as actigraphy and measurement of dim-light melatonin onset. ${ }^{21}$ This is unlikely to be a practical proposition in routine clinical settings at present.

We also need more information about the mechanism of action of agomelatine in terms of its putative interaction with $5-\mathrm{HT}_{2 \mathrm{C}}$ receptors and the most appropriate timing of administration in patients with depression. If agomelatine does indeed work through chronobiotic mechanisms, the timing of its administration in relation to the circadian cycle may be important. Hence the global instruction to administer the drug at 'bedtime' may not maximise its efficacy.

\section{About the authors}

Daniel Whiting is an Academic Clinical Fellow, and Philip J. Cowen is Professor of Psychopharmacology, University Department of Psychiatry, Warneford Hospital, Oxford.

\section{References}

1 Boivin DB. Influence of sleep-wake and circadian rhythm disturbances in psychiatric disorders. J Psychiatry Neurosci 2000; 25: 446-58.

2 Germain A, Kupfer DJ. Circadian rhythm disturbance in depression. Human Psychopharmacol 2008; 23: 571-85.

3 Pandi-Perumal SR, Trakht I, Spence DW, Srinivasan V, Dagan Y, Cardinali DP. The roles of melatonin and light in the pathophysiology and treatment of circadian rhythm sleep disorders. Nat Clin Pract Neurol 2008; 4: 436-47.

4 Millan MJ, Gobert A, Lejeune F, Dekeyne A, Newman-Tancredi A, Pasteau V, et al. The novel melatonin agonist agomelatine (S20098) is an antagonist at 5-hydroxytryptamine $2 \mathrm{C}$ receptors, blockade of which enhances the activity of frontocortical dopaminergic and adrenergic pathways. J Pharmacol Exp Ther 2003; 306: 954-64.

5 Pandi-Perumal SR, Trakht I, Srinivasan V, Spence DW, Masetroni GJM Zisapel N, et al. Physiological effects of melatonin: role of melatonin receptors and signal transduction pathways. Prog Neurobiol 2008; 85: 335-53.

6 San L, Arranz B. Agomelatine: a novel mechanism of antidepressant action involving the melatonergic and the serotonergic system. Eur Psychiatry 2008; 23: 396-402.
7 Tardito D, Molteni R, Popoli M, Racagni G. Synergistic mechanisms involved in the antidepressant effects of agomelatine. Eur Neuropsychopharmacol 2012; 22 (suppl 3): S482-6.

8 Molteni R, Calabrese F, Pisoni S, Gabriel C, Racagni G, Riva MA. Synergistic mechanisms in the modulation of the neurotrophin BDNF in the rat prefrontal cortex following acute agomelatine administration. World J Biol Psychiatry 2010; 11: 148-53.

9 Sen S, Duman R, Sanacora G. Serum brain-derived neurotrophic factor, depression, and antidepressant medications: meta-analyses and implications. Biol Psychiatry 2008; 64: 527-32.

10 Sharpley AL, Rawlings NB, Brain S, McTavish SFB, Cowen PJ. Does agomelatine block $5-\mathrm{HT}_{2 c}$ receptors in humans? Psychopharmacology 2011; 213: 653-5.

11 Krauchi K, Cajochen C, Mori D, Graw P, Wirz-Justice A. Early evening melatonin and S-20098 advance circadian phase and nocturnal regulation of core body temperature. Am J Physiol 1997; 272: R1178-88.

12 Leproult G, Van Onderbergen A, L'hermite-Balériaux M, Van Cauter E, Copinschi G. Phase-shifts of 24-h rhythms of hormonal release and body temperature following early evening administration of the melatonin agonist agomelatine in healthy older men. Clin Endocrino 2005; 63: 298-304

13 British Medical Association, Royal Pharmaceutical Society. British National Formulary (64th edn, September 2012). BMJ Group \& Pharmaceutical Press, 2012.

14 Hickie IB, Rogers N. Novel melatonin-based therapies: potential advances in the treatment of major depression. Lancet 2011; 378: 621-31.

15 Singh SP, Singh V, Kar N. Efficacy of agomelatine in major depressive disorder: meta-analysis and appraisal. Int J Neuropsychopharmacol 2012; 15: 417-28.

16 Barbui C, Cipriani A. Agomelatine and the brave old world of narrativebased medicine. Evid Based Ment Health 2012; 15: 2-3.

17 Rihmer Z, Dome P, Baldwin DS, Gonda X. Psychiatry should not become hostage to placebo: an alternative interpretation of antidepressantplacebo differences in the treatment response in depression. Eur Neuropsychopharmacol 2012; 22: 782-6.

18 Zajecka J, Schatzberg A, Stahl S, Caputo A, Post A. Efficacy and safety of agomelatine in the treatment of major depressive disorder: a multicentre, randomized, double-blind, placebo-controlled trial. J Clin Psychopharmacol 2010; 30: 135-44.

19 Montejo A, Majadas S, Rizvi SJ, Kennedy SH. The effects of agomelatine on sexual function in depressed patients and healthy volunteers. Hum Psychopharmacol 2011; 26: 537-42.

20 Sparshatt A, McAllister-Williams RH, Baldwin DS, Haddad PM, Bazire S, Weston $S$, et al. A naturalistic evaluation and audit database of agomelatine: clinical outcome at 12 weeks. Acta Psychiatr Scand 2013; 128: $203-11$.

21 Lewy AJ. Circadian misalignment in mood disturbances. Curr Psychiatry Rep 2009; 11: 459-65. 